\title{
Uptake of Rotavirus Vaccine and Factors That Contributed to Its Adoption and Acceptability by Parents/Guardians in Selected Communities of Ndola, Copperbelt Province, Zambia
}

\author{
Julia Shachakanza', Joseph Mumba Zulu², Margaret Maimbolwa3 \\ ${ }^{1}$ School of Medicine, Copperbelt University, Michael Chilufya Sata Campus, Ndola, Zambia \\ ${ }^{2}$ Health Promotion and Education Unit, Department of Public Health, School of Medicine, University of Zambia, Ridgeway \\ Campus, Lusaka, Zambia \\ ${ }^{3}$ Department of Nursing Science, School of Medicine, Ridgeway Campus, Lusaka, Zambia \\ Email: Juliashachakanza@yahoo.com, josephmumbazulu@gmail.com,mmaimbolwa@yahoo.com, \\ margaret.maimbolwa@unza.zm
}

How to cite this paper: Shachakanza, J., Zulu, J.M. and Maimbolwa, M. (2019) Uptake of Rotavirus Vaccine and Factors That Contributed to Its Adoption and Acceptability by Parents/Guardians in Selected Communities of Ndola, Copperbelt Province, Zambia. Health, 11, 415-427. https://doi.org/10.4236/health.2019.114037

Received: March 20, 2019

Accepted: April 25, 2019

Published: April 28, 2019

Copyright $\odot 2019$ by author(s) and Scientific Research Publishing Inc. This work is licensed under the Creative Commons Attribution-NonCommercial International License (CC BY-NC 4.0). http://creativecommons.org/licenses/by-nc/4.0/

\begin{abstract}
Rotavirus infection is recognized as a major cause of non-bacterial gastroenteritis infection affecting the stomach and intestinal tract in infants and young children worldwide leading to diarrhoea and vomiting. Most of the children would have had rotavirus infection by the time they are five years old. The disease is highly contagious and unpredictable as it can lead to dehydration due to severe loss of body fluid and consequently to death. World Health Organization recommended the use of two vaccines, RotaTeq ${ }^{\mathrm{R}}$ and Rotarix $^{\mathrm{TM}}$ to be used in countries with high diarrhoea related mortality in under-five children. In Zambia rotavirus vaccine (Rotarix ${ }^{\mathrm{TM}}$ ) was rolled out to other parts of the country in its immunization program on 27th November 2013 after a successful pilot study in Lusaka in 2012 to reduce on diarrhoea caused by rotavirus infection among under-five children which had caused $9 \%$ of deaths. The objectives for this study were to establish uptake of rotavirus vaccine among under-fives and factors that shaped its adoption and acceptability at community level in Ndola, Zambia. Study design was cross sectional analytic community based which was conducted from 1st March 2017 to 28th September 2017 to compare factors that contributed to its uptake. Sample size consisted of 380 respondents who were parents/guardians to under-five children aged 3 - 36 months residing in two homogenous selected communities. Purposive sampling was used to select study sites and respondents. This paper used triangulation method of data collection which included checklist, structured interview schedule and discussion guide tools.
\end{abstract}


Statistical Package for Social Science (SPSS) was used for quantitative data analysis and Atlas.ti for qualitative data obtained from Focus Group Discussions (FGD) using hermeneutics theoretical frames to identify linkages and associations of variables. Results have shown that majority (85.3\%) of under-fives received two recommended rotavirus vaccine (high uptake). Under-fives aged between 13 - 18 months were 11.32 times more likely to receive high uptake of rotavirus vaccine than other age groups. Availability of rotavirus vaccine at health facilities $(\mathrm{p}=0.035)$ contributed to its adoption and acceptability. In conclusion, availability of rotavirus vaccine at health facilities had an impact on its uptake and adoption.

\section{Keywords}

Acceptability, Adoption, Perception, Rotavirus Vaccine, Uptake

\section{Introduction}

Rotavirus causes high rates of morbidity and mortality among under-five children throughout the world. When rotavirus infection enters the body through oral route, it infects and damages the gastro-intestinal tract cells making it difficult for absorption of water and nutrients by the body which may be fatal [1]. The infection is mainly characterised by vomiting, fever, diarrhoea and loss of appetite. The incubation period is $1-3$ days. Symptoms begin abruptly and disappear within 3 - 7 days [2]. The term rotavirus is derived from a Latin word "rota" meaning "wheel-like" appearance of the virus when viewed by electron microscope [3]. There are five species of this virus namely: A, B, C, D and E. Among these types, rotavirus $A$ is the most common cause of more than $90 \%$ of the infections in humans and $20 \%$ fatality in young children [4]. In the same report, diarrhoea was cited to be the leading cause of childhood mortality contributing to approximately 1.87 million deaths each year in developing countries. Hence, the United Nations developed the Millennium Development Goal (MDG) number four to reduce the mortality rate among under-five children in developing countries by two-thirds between 1990 and 2015 [4]. To combat severe diarrhoea caused by the rotavirus infection, a rotavirus vaccine was introduced to mitigate the infection. Therefore, this paper assessed under-five children's uptake of rotavirus vaccine and factors that contributed to its acceptability and adoption by parents/guardians.

A cohort study conducted on infants in the United States by Panozzo [5] revealed that about $69 \%(594,117)$ eligible children received the first dose of the rotavirus vaccine from 2006-2010. The majority of children received the vaccine at the recommended age. More children were seen to have received monovalent rotavirus vaccine than pentavalent rotavirus vaccine or a mix of the vaccines, $87 \%, 79 \%$ and $73 \%$ respectively $(\mathrm{p}<0.001)$.

A retrospective 2012 survey conducted by Braeckman [6] in Flanders, Belgium 
among infants born in 2010 revealed that children received $31.7 \%$ and $10.1 \%$ of their first and second dose of rotavirus vaccine at the recommended age respectively. In this study, 874 families were interviewed at their homes using a standardized questionnaire. Factors cited to have contributed to non-vaccination of the eligible children included unemployment of mother and non-availability of the vaccine at some point in time. The other reason was that the two vaccines Rotarix $^{\mathrm{TM}}$ (two-dose schedule) and Rotateq ${ }^{\mathrm{R}}$ (three-dose schedule) were only available in private pharmacies which were not freely given.

A study conducted in the United States Medicaid, recipients [7] revealed low rates of unvaccinated children (40\%) despite CDC recommendations on the need for under-five children to receive vaccinations. Orozco's study conducted in Nicaragua [8] revealed that the new rotavirus vaccine was introduced in October 2006. The results revealed that 1 - dose vaccine coverage rate increased very rapidly after the introduction of RotaTeq ${ }^{\mathrm{R}}$ in 2007 . The uptake of the $2^{\text {nd }}$ and $3^{\text {rd }}$ dose vaccine among age groups 0 - 11 months was $26 \%$.

Dube's study conducted in Canada between 2008 and 2009 [9] indicated that most $(67 \%)$ parents accepted to have their children immunized due to parental intentions, personal beliefs, moral correctness of their children being immunized, significant others' approval and household income. The conclusion of the study was that acceptability of rotavirus vaccinations was dependent on health promotion which addressed parental knowledge, attitudes and beliefs regarding the disease and the vaccine to be given. Another study conducted in the Democratic Republic of Congo [10] revealed that in spite of the misconception of the acute watery diarrhoea disease being caused by witchcraft $(5.1 \%)$ and breastfeeding (4.8\%), anticipated acceptability of the rotavirus vaccine was at $99 \%$ if no cost was attached to its administration. In the event that cost was attached to the vaccination, educational level of the parents was a major predictor. In addition, acceptability was high if the vaccines were incorporated in the existing national immunization schedule.

A study conducted by Gargano [11] from 2009 to 2010 to predict physicians' perception to identify the predictors of administration and attitude toward vaccines which included rotavirus, revealed that only one tenth of Pediatricians had been routinely administering rotavirus vaccines in India. On the contrary, Nnenna's study conducted [12] in Nigeria revealed that health care providers were more likely to recommend their clients to use the vaccine if it was incorporated in the National Programme of Immunization $(72.5 \%)$ or recommended by Paediatrics Association of Nigeria (70.3\%) or the Federal Ministry of Health (69.2\%). About $70.3 \%$ of respondents stated that they would have their own children vaccinated if provided freely and only $2.2 \%$ mentioned that would not. Despite the stated acceptance levels by the parents/guardians, $29 \%$ of them expressed some concern on the new vaccine, $18 \%$ on the live nature of the vaccine while $16 \%$ were concerned on how safe the vaccine would be to their children after its administration. On the other hand, $7.7 \%$ were concerned about the side effects of the vaccine. 
WHO and UNICEF [13] recommended the use of the two live oral attenuated vaccines against rotavirus infection which were licensed and approved for use globally which included Rotarix ${ }^{\mathrm{TM}}$ (manufactured by GlaxoSmithKline) and $\operatorname{RotaTeq}^{\mathrm{R}}$ (manufactured by Merck). These vaccines were recommended as an important measure and the most cost effective public health intervention to reduce deaths due to severe rotavirus-associated diarrhoea. In Zambia Rotarix ${ }^{\mathrm{TM}}$ vaccine is used and administered as a two-dose regimen the first of which is given from 6 weeks to 15 weeks of child's age while the second from 10 weeks to 32 weeks of age.

Zambia became the 18th GAVI-eligible country on the $27^{\text {th }}$ of November 2013 to introduce rotavirus vaccine (Rotarix ${ }^{\mathrm{TM}}$ ) in its immunization programme for prevention of diarrhoea, childhood deaths and hospitalizations among under-five children. The programme was combined with community projects to teach mothers to stop children from getting diarrhoea [14] [15]. According to Health Management Information System [16] statistics from Ndola District Health Office on the Copperbelt Province in Zambia have shown a high coverage of vaccinations at the health facilities among the eligible under-five children which is above the target of $85 \%$.

\section{Methods}

A cross sectional community based analytical study was used to determine background informationon uptake of rotavirus vaccine, its adoption and acceptability at community level. The study was cross sectional because it covered a specified age group between 3 - 36 months at one point in time to establish rotavirus uptake and factors that contributed to its adoption and acceptability by parents/guardians. Study settings included two homogenous communities serviced by New Masala and Chipokota Mayamba health facilities. Sample size was 380 which included under-five children aged 3 - 36 months whose respondents were parents/guardians from the households. The sample size was adequate and arrived at using the formula: $\mathrm{N}=\mathrm{z}^{2} \mathrm{PQ} / \mathrm{d}^{2}$ to represent the given population. Purposive sampling was used to select the two communities, health care providers and parents/guardians. Health care providers included nurses and midwives working at the selected health facilities. Checklists were used for data collection from health care providers while structured interview schedule and interview guides were used to collect data from parents/guardian. A checklist was used to assess the availability of rotavirus vaccine at the health facilities. Under-five children's clinic cards were used to verify type and number of times child received rotavirus vaccine. Quantitative data collection was conducted using structured face to face interviews to generate numerical data from households visited. Variables used were gender, age, messages received on hygiene and sanitation, education level, occupation, income, availability of rotavirus vaccine, adoption, acceptability and knowledge by uptake of rotavirus vaccine. SPSS version 24 software was used for data entry and analysis of quantitative data which was numerical in nature. Pearson's Chi-square was used in a bivariate regression 
to determine relationships and associations between two nominal variables and Fisher's Exact Significant test in a $2 \times 2$ table with values less than five. In instances where the Chi-square results were statistically significant further analysis using backward logistic regression was used to determine associations between these variables and uptake of rotavirus vaccine.

Qualitative data was obtained using unstructured discussion guide to gain understanding of underlying reasons, opinions and expressions from respondents to compliment quantitative data. A total of twenty group discussions called Focus Group Discussions (FGDs) each comprising ten respondents selected purposively were conducted at outreach sites within the two selected communities. Data from FGDs was transcribed in Microsoft word in form of verbatim. The transcripts were imported into Atlas.ti version 8 statistical package as primary data (PD). The data was categorized, themes identified and linkages developed. The themes included experiences, misconceptions (beliefs/religion), practices and health impact.

\section{Limitation of the Study}

The study based its findings from household respondents who could have had memory bias in retrieval of information during the collection of data. Laboratory investigations were not included in this part of the study as its concentration was on rotavirus uptake, its adoption and acceptability by respondents.

\section{Results}

The total number of respondents enrolled were 380 which included the parents/guardians to under-five children aged 3 - 36 months. Study findings revealed that majority $324(85.3 \%)$ of under-five children received the recommended dose of rotavirus vaccine twice as per national guideline for the control of diarrhoea caused by rotavirus infection. Study results have also revealed that majority of respondents were aged between 25 and 34 years 178 (46.8\%) followed by those 150 (39.5\%) aged 15 - 24 years. Study revealed that majority 273 $(71.8 \%)$ of respondents were married. Among the 273 of those that were married, majority $236(72.8 \%)$ of their children had received high uptake of rotavirus vaccine. Study results revealed that despite majority of respondents $289(76.1 \%)$ having attained secondary education only $3.9 \%$ reached college/tertiary education. In terms of occupation about half 195 (51.3\%) of respondents were housewives while only 114 (30\%) were either in formal or informal employment. More than half of respondents 205 (53.9\%) had an income of K1000 and below which was not enough to sustain them throughout the month hence respondents depended on their husbands for financial support. Results have revealed that out of $362(95.3 \%)$ respondents who had perceived rotavirus vaccine to be effective in the prevention of diarrhoea among under-fives, majority 311 (96\%) received high uptake of rotavirus vaccine. In spite of these disparities, there was no significant statistical association between respondents' demographic data and knowledge on rotavirus infection in relation to uptake of rotavirus vaccine 
among under-five children (Table 1).

Results from FGDs perceived efficacy of rotavirus vaccine differently. For instance, R9, (FGD 13) said, "I think that vaccines work because my child has never had diarrhoea for a long time now". Many others admitted that vaccinated children if suffered from diarrhoea, it was very mild and that recovery occurred within a short period of time. Other respondents mentioned that the vaccine did not work. For instance, one said, "What I think is that vaccine does not work because my child had diarrhoea which was severe in the last two months," (R8, FGD 2). Another respondent argued saying, "I feel sometimes vaccines do not work because children still suffer from diarrhoea when teething," (R5, FGD 16) (Figure 1).

When respondents were asked for their opinion on whether the community accepted rotavirus vaccine more than half of them $228(60 \%)$ said "Yes" while $127(33.4 \%)$ said "No" $(p=0.386)$. These results show no significant statistical association between opinion and uptake of rotavirus vaccine. Some reasons given by respondents as to why community did not accept rotavirus vaccine almost half of them 68 (53.5\%) stated that they had no idea while others mentioned laziness 47 (37\%). FGD results revealed that culture and religious beliefs were seen to be affecting acceptability of vaccines among respondents. One respondent mentioned, "Some mothers do not accept vaccinations because they say that it is only God who protects a child from getting sick. Some say, 'Abana bakula fye' in Bemba tribe meaning [children just grow up naturally]", (R5, FGD 14).

One interesting perceived contributing factor to diarrhoea in FGDs was a condition called "nalukungu" in Bemba, translated to mean pimple on upper palate of child's mouth (R1, FGD 3). The other factor that was cited was the growth process that children undergo as being unique. Majority of respondents mentioned teething as a major contributing factor to diarrhoea among under-five children. One respondent said that diarrhoea during teething was normal. According to her,

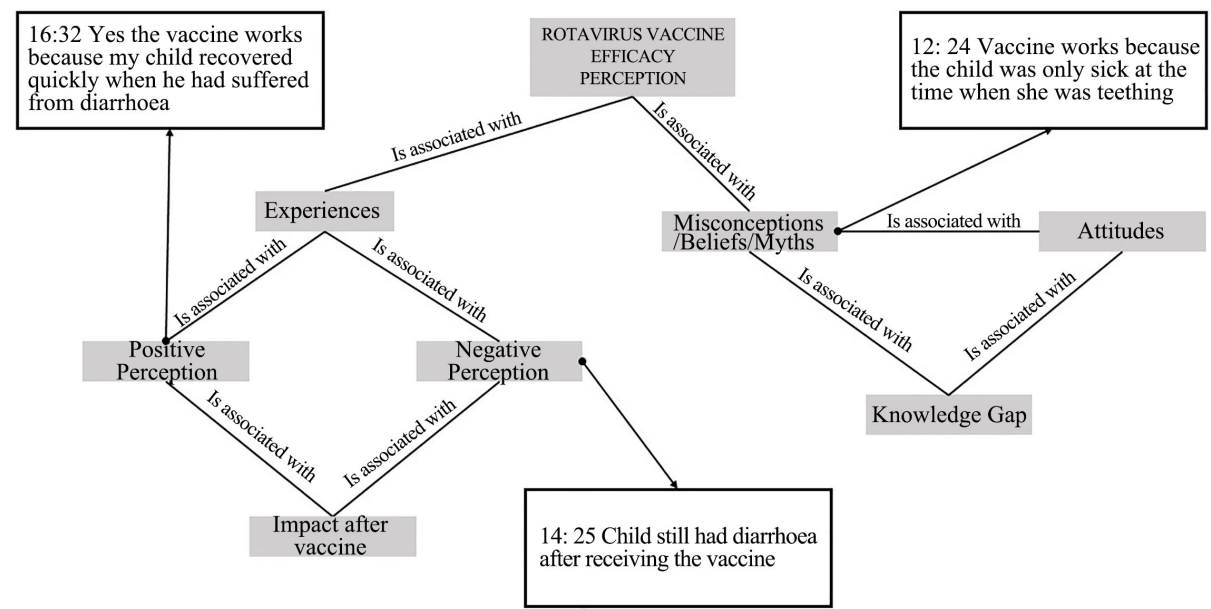

Figure 1. FGD respondent's perceived perception on efficacy of rotavirus vaccine and its factors. 
Table 1. Respondents'demographic characteristics and knowledge by uptake of rotavirus vaccine.

\begin{tabular}{|c|c|c|c|c|c|}
\hline \multirow{2}{*}{ Characteristics } & \multirow{2}{*}{$\begin{array}{c}\text { Total = } 380 \\
\mathrm{~N}(\%)\end{array}$} & \multicolumn{2}{|c|}{ Uptake of rotavirus vaccine } & \multirow{2}{*}{\multicolumn{2}{|c|}{$\mathrm{X}^{2} \mathrm{p}$-value }} \\
\hline & & Low $=56$ & High $=324$ & & \\
\hline \multicolumn{6}{|c|}{ Age } \\
\hline $15-24$ years & $150(39.5)$ & $25(44.6)$ & $125(38.6)$ & 0.78 & 0.854 \\
\hline 25 - 34 years & $178(46.8)$ & $24(42.9)$ & $154(47.5)$ & & \\
\hline 35 - 44 years & $43(11.3)$ & $6(10.7)$ & $37(11.4)$ & & \\
\hline 45 and above years & $9(2.4)$ & $1(1.8)$ & $8(2.5)$ & & \\
\hline \multicolumn{6}{|c|}{ Marital status } \\
\hline Single & $107(28.2)$ & $19(33.9)$ & $88(27.2)$ & & $0.335^{\mathrm{a}}$ \\
\hline Married & $273(71.8)$ & $37(66.1)$ & $236(72.8)$ & & \\
\hline \multicolumn{6}{|c|}{ Educational level } \\
\hline College/University & $15(3.9)$ & $0(0)$ & $15(4.6)$ & 4.47 & 0.107 \\
\hline Secondary & $289(76.1)$ & $48(85.7)$ & $241(74.4)$ & & \\
\hline Primary & $76(20.0)$ & $8(14.3)$ & $68(21.0)$ & & \\
\hline \multicolumn{6}{|c|}{ Occupation } \\
\hline Housewife & $195(51.3)$ & $27(48.2)$ & $168(51.9)$ & 2.98 & 0.226 \\
\hline Dependent/student & $71(18.7)$ & $15(26.8)$ & $56(17.2)$ & & \\
\hline Formal/informal employment & $114(30.0)$ & $14(25.0)$ & $100(30.9)$ & & \\
\hline \multicolumn{6}{|l|}{ Income } \\
\hline Do not know & $48(12.6)$ & $10(17.9)$ & $38(11.7)$ & 4.57 & 0.334 \\
\hline 1000 and below & $205(53.9)$ & $25(44.6)$ & $180(55.6)$ & & \\
\hline $1001-2000$ & $72(18.9)$ & $12(21.4)$ & $60(18.5)$ & & \\
\hline $2001-3000$ & $26(6.8)$ & $6(10.7)$ & $20(6.2)$ & & \\
\hline Above 3000 & $29(7.6)$ & $3(5.4)$ & $26(8.0)$ & & \\
\hline \multicolumn{6}{|c|}{ Knowledgeable on rotavirus infection } \\
\hline Yes & $47(12.4$ & $8(14.3)$ & $39(12.0)$ & 0.22 & 0.637 \\
\hline No & $333(87.6)$ & $48(85.7)$ & $285(88.0)$ & & \\
\hline \multicolumn{6}{|c|}{ Perception on whether vaccines work in diarrhoea prevention } \\
\hline Yes & $362(95.3)$ & $51(91.1)$ & $311(96.0)$ & 2.65 & 0.266 \\
\hline No & $3(0.8)$ & $1(1.8)$ & $2(0.6)$ & & \\
\hline Do not know & $15(3.9)$ & $4(7.1)$ & $11(3.4)$ & & \\
\hline \multicolumn{6}{|c|}{ Reasons on perception opinion on whether vaccine work in diarrhoea prevention } \\
\hline Suffered from diarrhoea & $3(0.8)$ & $1(1.8)$ & $2(0.6)$ & 3.42 & 0.332 \\
\hline Do not suffer & $180(47.4)$ & $23(41.1)$ & $157(48.5)$ & & \\
\hline Diarrhoea reduces & $168(44.2)$ & $25(44.6)$ & $143(44.1)$ & & \\
\hline Do not know & $29(7.6)$ & $7(12.5)$ & $22(6.8)$ & & \\
\hline
\end{tabular}

${ }^{\star}$ Pearson Continuity Correction, ${ }^{a}$ Fishers Exact Test. 
"What I know is that a child usually has diarrhoea at the time when teeth are about to show up from the gums and I know that most children do suffer from diarrhoea during this time", (R10, FGD 6).

Weather was believed to have an effect on children's diarrhoea. The effect of heat on food and water could not go unmentioned among respondents. According to one respondent, heat itself comes with a lot of diseases because it makes the water warm and that the more the child feels thirst the more the desire to drink water resulting in diarrhoea.

Results from health care providers' checklist revealed that 25 (83.3\%) received no reports on adverse effects from respondents after their children received the vaccine. Majority 28 (93.3\%) of health care providers mentioned having an updated micro plan for routine immunizations, specified target population, adequate rotavirus vaccines and registers used for accountability of vaccinations to ensure no shortage of the vaccine at health facilities. Study results revealed a significant statistical association between availability of rotavirus vaccine at health facilities and its uptake ( $\mathrm{p}=0.035)$. Respondents who mentioned "Yes" rotavirus was available at health facilities were 2.18 times more likely to receive high uptake of rotavirus vaccine than those who mentioned "No" (Table 2).

Multivariate analysis logistic regression results revealed significant statistical association between age group visited, feeding option, duration of breast/artificial feeds and messages received on hygiene and sanitation by uptake of rotavirus vaccine. Children visited aged between 13 - 18 months were 11.32 times more likely to have high uptake of rotavirus vaccine as recommended by national health policy while those aged between 25 - 30 months were 3.28 times to receive high uptake than other age groups. Under-fives who were on breast milk as a feeding option were 2.05 times more likely to have high uptake of the recommended dose of rotavirus vaccine than those that were artificially fed. In addition, under-five children who were still on breast/artificial feeds were 2.63 times more likely to have high uptake of rotavirus vaccine where as those who breast fed for 6 - 12 months were 0.5 times less likely to have high uptake of rotavirus vaccine. Furthermore, study results have revealed that inspite of respondents having received messages on hygiene and sanitation, they were 0.54 times $(1-0.46=0.54)$ less likely to adhere to the two recommended dose regimen of rotavirus vaccine as per national guidelines compared to those that had not received health messages (Table 3 ).

Table 2. Availability of rotavirus at health facilities by its uptake.

\begin{tabular}{|c|c|c|c|c|}
\hline Characteristics & p-value & Odds Ratio (OR) & \multicolumn{2}{|c|}{$95 \%$ CI } \\
\hline \multicolumn{5}{|c|}{ Availability of rotavirus vaccine at health facility } \\
\hline Yes & 0.035 & 2.18 & 1.055 & 4.511 \\
\hline
\end{tabular}

Backward Logistic Regression, CI = Confidence Interval. 
Table 3. Age, feeding optionand messages received by rotavirus vaccine uptake.

\begin{tabular}{|c|c|c|c|}
\hline Characteristics & p-value & Odds Ratio (OR) & $95 \% \mathrm{CI}$ \\
\hline \multicolumn{4}{|l|}{ Child's age } \\
\hline 6 months \& below & 0.734 & 0.73 & $0.12,4.57$ \\
\hline $7-12$ months & 0.569 & 1.63 & $0.30,8.99$ \\
\hline $13-18$ months & 0.004 & 11.32 & $2.20,58.17$ \\
\hline $19-24$ months & 0.187 & 1.97 & $0.72,5.41$ \\
\hline 25 - 30 months & 0.033 & 3.28 & $1.10,9.80$ \\
\hline \multicolumn{4}{|l|}{$31-36$ months (Reference) } \\
\hline \multicolumn{4}{|c|}{ Type of child's feeding option } \\
\hline Breast fed & 0.051 & 2.05 & $2.0,4.23$ \\
\hline \multicolumn{4}{|l|}{ Artificially fed (Reference) } \\
\hline \multicolumn{4}{|c|}{ Duration of child's breast/artificial feeding } \\
\hline Still on breast/artificial feeds & 0.017 & 2.63 & $0.41,16.61$ \\
\hline $6-12$ months & 0.028 & 0.50 & $0.16,1.60$ \\
\hline $13-18$ months & 0.958 & 1.53 & $0.55,4.29$ \\
\hline \multicolumn{4}{|l|}{ Above 18 months (reference) } \\
\hline \multicolumn{4}{|c|}{ Messages received on hygiene and sanitation } \\
\hline Yes & 0.048 & 0.46 & $0.22,0.99$ \\
\hline No (Reference) & & & \\
\hline
\end{tabular}

Binary and Multivariate Logistic Regression, $\mathrm{CI}=$ Confidence Interval.

\section{Discussion}

The sample size in this study was 380 under-five children aged 3 - 36 months whose respondents were female parents/guardians. This age group was considered because it fell within the ages that started receiving the rotavirus vaccine when it was first rolled out in Zambia on $27^{\text {th }}$ November 2013. Rotavirus vaccine is recommended worldwide as a policy in prevention of diarrhoea disease caused by rotavirus infection. Results have shown that children from respondents aged 25 - 34 years and 15 - 24 years received high uptake of rotavirus vaccine of $47.5 \%$ and $38.6 \%$ respectively. In addition, more than half $(71.8 \%)$ of respondents were married while $28.2 \%$ were single. The single included those that were not married, widowed and divorced.

Study results have revealed that majority $(76.1 \%)$ of respondents reached secondary education level and only 3.9\% reached college/university level. Results have also shown that half of respondents were housewives $(51.3 \%)$ hence they depended on their spouses for financial support. Only $30 \%$ of respondents were in formal/informal employment. Results in this study have revealed that slightly above half (53.9\%) of respondents had an income of K1000 and below. In spite of the above disparities, results have revealed no significant statistical association between respondents' age, marital status, educational level, occupation and in- 
come in relation to uptake of rotavirus vaccine. Furthermore, this study has shown that majority of respondents had no knowledge on rotavirus infection (87.6\%). Results have also shown that respondents' perception on efficacy of rotavirus vaccine in diarrhoea prevention was good. Majority of them mentioned that children who received rotavirus vaccine did not suffer from diarrhoea (47.4\%) followed by those (44.2\%) who said that even if a vaccinated child suffered from diarrhoea the condition was less likely to be severe compared to those who were not vaccinated. However, the results showed no significant statistical association between knowledge on rotavirus infection, perception on efficacy of the vaccine in diarrhoea prevention and reasons given for their opinion in relation to uptake of rotavirus vaccine.

Results have shown that majority (85.3\%) of under-fives received the two recommended rotavirus vaccine for the control of diarrhoea diseases caused by rotavirus infection. This study confirms statistics [16] obtained from Ndola District Health Office on uptake coverage of $85 \%$. This study's results were similar to a study conducted by Braeckman [6] which revealed that majority of children received the vaccines at the recommended age. Factors cited to have contributed to non-vaccination of the eligible children included unemployment of mother and the cost of the vaccine in private pharmacies which is not the case in Zambia as vaccines are provided to under-fives at no cost. Study results revealed significant statistical association between availability of rotavirus vaccine at health facilities and its uptake ( $\mathrm{p}=0.035)$. Majority $(88.9 \%)$ of respondents who answered "Yes vaccine was available" had high uptake of rotavirus vaccine than the $11.1 \%$ who mentioned that vaccine was not available. Further analysis using multivariate logistic regression results revealed that respondents who mentioned that rotavirus vaccine was available at health facility were 2.18 times more likely to have a high uptake of rotavirus vaccine than those who said vaccines were not available. On the contrary, study results conducted by Braeckman in Flanders of Belgium [6] revealed that non-vaccination of the eligible children was due to non-availability of rotavirus vaccine at some point in time.

Study conducted in the United States Medicaid [7] revealed low rates of unvaccinated children (40\%) despite CDC recommendations on the need for under-five children to receive vaccinations. Orozco's study results conducted in Nicaragua [8] revealed that the new rotavirus vaccine was introduced in October 2006. The results revealed that 1 -dose vaccine coverage rate increased rapidly after introduction of RotaTeq ${ }^{\mathrm{R}}$ in 2007 . The uptake of the $2^{\text {nd }}$ and $3^{\text {rd }}$ dose vaccine among age group 0 - 11 months was $26 \%$. In this study, results have revealed that children visited aged between 13 - 18 months were 11.319 times more likely to receive high uptake of rotavirus vaccine while those aged 25 - 30 months were 3.281 times than other age groups as recommended by national health policy. This clearly indicates that parents/guardians are more actively involved in taking their children for vaccination up to the age of one and half years when they receive the booster dose of other vaccines. Study results have revealed 
that under-fives who were on breast milk as a feeding option and still on breast/artificial feeds were about 2 times more likely to receive high uptake of rotavirus vaccine as per national guidelines. This simply shows that rotavirus vaccine is given to children in their early infancy hence the high uptake in this age group. This study has revealed that in spite of respondents having received messages on hygiene and sanitation, they were 0.54 times less likely to adhere to the two dose vaccine regimen as per national guideline compared to those who had not. This simply means that respondents took their children for vaccination as a routine activity as recommended by health care providers at their various health facilities.

On contrary, Dube's study findings [9] in Canada between 2008 and 2009 revealed that acceptability of rotavirus vaccine was dependent on health promotion which addresses knowledge, attitudes and beliefs regarding diseases and vaccine to be given to the child. In this study, more than half of respondents who mentioned having received health messages on hygiene and sanitation cited health care providers as the major source of information, while less than halfcited community health workers. This means that there must be combined efforts to provide hygiene and sanitation messages from all stake holders from other Ministries.

Study results revealed that good perception on rotavirus vaccine efficacy contributed to its acceptability. Majority of respondents (96\%) mentioned that rotavirus vaccine works in diarrhoea prevention because children do not suffer from diarrhoea and that its severity reduces when child is vaccinated. These results were not any different from FGD results. Majority of respondents (98.9\%) had not heard about some myths concerning rotavirus vaccine from the communities where they lived. Some myths cited although insignificant in this study included body hotness, skin rash, vaccines do not work and that eyes became weak after the child had been vaccinated. Only a few respondents from FGDs indicated, "Abana bakula fye" meaning children just "grow up naturally" even without the vaccine, a term used in "Bemba tribe" while others said children just grow up with air around the atmosphere.

Results obtained from health care providers revealed that majority (83.3\%) mentioned that there were no adverse effects received on rotavirus vaccine from parents/guardians of children after vaccination sessions. The findings in this study were similar to those [12] in Nigeria, which revealed insignificant side effects of the vaccine. This study has also revealed that majority (83.3\%) of health care providers conducted growth monitoring programs at outreach sites in the last week of each month according to plan. These programs help in improving the coverage of immunizations for disease prevention.

\section{Conclusion}

This study has revealed that availability of rotavirus vaccine and its inclusion in the existing national guidelines shaped its adoption and acceptability by par- 
ents/guardians.

\section{Acknowledgements}

We are grateful for the understanding and participation of all respondents, staff, sponsors for the Combi stripes for testing of rotavirus and adenovirus in stool.

\section{Declaration}

I Julia Shachakanza, do hereby declare that this work is our own and that the work of other persons utilized in this dissertation has been duly acknowledged. This work presented here has not been previously presented at this or any other university for similar purposes.

\section{Approval}

Approval was obtained from Research Ethics Committee at Tropical Disease Research Centre (TDRC). Permission was sought from Ministry of Health $(\mathrm{MoH})$, Ndola District Health Management Office and Provincial Health Office. Respondents were assured of confidentiality and non-persecution arising from their responses. No names were written on the questionnaires concerning their responses.

\section{Funding}

This research did not receive any specific grant from funding agencies in the public, commercial, or not-for-profit sectors.

\section{Conflicts of Interest}

The authors declare no conflicts of interest regarding the publication of this paper.

\section{References}

[1] Artherly, D.E. (2012) Projected Health and Economic Impact of Rotavirus Vaccination in GAVI-Eligible Countries: 2011-2030. Vaccines, 30, A7-A14. https://doi.org/10.1016/j.vaccine.2011.12.096

[2] MCD MCH (2013) Rotavirus Vaccination Handbook. A Training Manual for Health Workers, Lusaka.

[3] Mameli, C. (2012) New Insights into Rotavirus Vaccines. Human Vaccines and Immunotherapeutics, 8, 1022-1028. https://doi.org/10.4161/hv.20295

[4] GAVI Alliance (2013) Rotavirus Disease. Geneva.

[5] Panozzo, C. (2013) Patterns of Rotavirus Vaccine Uptake and Use in Privately-Insured US Infants, 2006-2010. PLoS ONE, 8, e73825. https://doi.org/10.1371/journal.pone.0073825

[6] Braeckman, T. (2014) Rotavirus Vaccination Coverage and Adherance to Recommended Age among Infants in Flanders, Belgium in 2012. Euro Surveillance, 19, 1560-7917. https://doi.org/10.2807/1560-7917.ES2014.19.20.20806

[7] Krishnarajah, G. (2014) Rotavirus Vaccination Compliance and Completion in Me- 
dicaid Infant Population. Vaccine in United States, 33, 479-486.

https://doi.org/10.1016/j.vaccine.2014.06.059

[8] Orozco, M. (2015) Uptake of Rotavirus Vaccine and National Trends of Acute Gastroenteritis among Children in Nicaragua. Oxford Journals, Medicine and Health Journal of Infectious Diseases, 200, S125-S130. https://doi.org/10.1086/605053

[9] Dube, E. (2012) Determinants of Parents' Decision to Vaccinate Their Children against Rotavirus: Results of a Longitudinal Study. Oxford Journals Health Education Research, 10, 1-12. https://doi.org/10.1093/her/cys088

[10] Merten, S. (2013) Social Cultural Determinants of Anticipated Vaccine Acceptance for Acute Watery Diarrhoea in Early Childhood in Katanga Province, Democratic Republic of Congo. American Journal Tropical Medicine Hygiene, 89, 419-425. https://doi.org/10.4269/ajtmh.12-0643

[11] Gargano, L.M. (2012) Predictors of Administration and Attitudes about Pneumococcal, Haemophilus Influenzae Type B and Rotavirus Vaccines among Pediatricians in India: A National Survey. Vaccine, 30, 3541-3545. https://doi.org/10.1016/j.vaccine.2012.03.064

[12] Nnenna, T.B. (2013) Knowledge of Rotavirus among Health Care Providers and Their Acceptance of Rotavirus in South-East, Nigeria. Journal of Vaccines \& Vaccination, S1, 005. https://doi.org/10.4172/2157-7560.S1-005

[13] World Health Organization and UNICEF (2013) Ending Preventable Child Deaths from Pneumonia and Diarrhoea by 2025: The Integrated Global Action Plan for Pneumonia and Diarrhoea. WHO, Brazzaville.

[14] PATH, GAVI Alliance (2013) Rotavirus Infection. Geneva.

[15] World Health Organization (2015) Report on WHO Launches African Vaccination Week in Zambia. Lusaka.

[16] Ndola District Health Management Team (2015) Health Management Information System, 2011-2015. Ndola. 